\title{
Probing New Long-Range Interactions by Isotope Shift Spectroscopy
}

\author{
Julian C. Berengut, ${ }^{1, *}$ Dmitry Budker, ${ }^{2,3,4, \dagger}$ Cédric Delaunay, ${ }^{5,}$ Victor V. Flambaum, ${ }^{1, \S}$ Claudia Frugiuele, ${ }^{6, \|}$ \\ Elina Fuchs, ${ }^{6,4}$ Christophe Grojean, ${ }^{7,8, * *}$ Roni Harnik, ${ }^{9, \dagger}$ Roee Ozeri, ${ }^{10, *}$ Gilad Perez, ${ }^{6,8}$ and Yotam Soreq ${ }^{11,\|\|}$ \\ ${ }^{1}$ School of Physics, University of New South Wales, Sydney, New South Wales 2052, Australia \\ ${ }^{2}$ Helmholtz-Institut Mainz, Johannes Gutenberg-Universität Mainz, 55128 Mainz, Germany \\ ${ }^{3}$ Physics Department, University of California, Berkeley 94720-7300, USA \\ ${ }^{4}$ Nuclear Science Division, Lawrence Berkeley National Laboratory, Berkeley, California 94720, USA \\ ${ }^{5}$ Laboratoire d'Annecy-le-Vieux de Physique Théorique LAPTh, CNRS-Université Savoie Mont Blanc, \\ BP 110, F-74941 Annecy-le-Vieux, France \\ ${ }^{6}$ Department of Particle Physics and Astrophysics, Weizmann Institute of Science, Rehovot 7610001, Israel \\ ${ }^{7}$ DESY, D-22607 Hamburg, Germany \\ ${ }^{8}$ Institut für Physik, Humboldt-Universität zu Berlin, D-12489 Berlin, Germany \\ ${ }^{9}$ Theoretical Physics Department, Fermi National Accelerator Laboratory, Batavia, Illinois 60510, USA \\ ${ }^{10}$ Department of Physics of Complex Systems, Weizmann Institute of Science, Rehovot 7610001, Israel \\ ${ }^{11}$ Center for Theoretical Physics, Massachusetts Institute of Technology, Cambridge, Massachusetts 02139, USA
}

(Received 22 May 2017; revised manuscript received 20 September 2017; published 26 February 2018)

We explore a method to probe new long- and intermediate-range interactions using precision atomic isotope shift spectroscopy. We develop a formalism to interpret linear King plots as bounds on new physics with minimal theory inputs. We focus only on bounding the new physics contributions that can be calculated independently of the standard model nuclear effects. We apply our method to existing $\mathrm{Ca}^{+}$data and project its sensitivity to conjectured new bosons with spin-independent couplings to the electron and the neutron using narrow transitions in other atoms and ions, specifically, $\mathrm{Sr}$ and $\mathrm{Yb}$. Future measurements are expected to improve the relative precision by 5 orders of magnitude, and they can potentially lead to an unprecedented sensitivity for bosons within the 0.3 to $10 \mathrm{MeV}$ mass range.

DOI: 10.1103/PhysRevLett.120.091801

Introduction.-The quest for new physics (NP) beyond the standard model (SM) of particle physics is pursued in multiple directions. Current efforts with colliders such as the LHC form the so-called energy frontier, probing directly the $\mathrm{TeV}$ energy scale. Other accelerators, such as meson factories, beam dump, and neutrino experiments, form the intensity frontier that broadly explores the $\mathrm{MeV}-\mathrm{GeV}$ scale. Atomic physics tabletop experiments form a third frontier of precision measurements (see, e.g., Refs. [1-5]; for a review, see Refs. [6-8]) where sub-MeV physics can be efficiently tested. It is interesting to note that NP that may account for the hierarchy issues could be new light scalars that couple to matter fields [9-15]. To convert the high precision offered by atomic and molecular spectroscopy into sensitivity to a fundamental new physics, one either has to acquire a similar theoretical accuracy of atomic structure or, alternatively, search for unique observables that are insensitive to theoretical uncertainties.

Published by the American Physical Society under the terms of the Creative Commons Attribution 4.0 International license. Further distribution of this work must maintain attribution to the author(s) and the published article's title, journal citation, and DOI. Funded by SCOAP .
In this Letter we show that precision isotope shift (IS) spectroscopy may probe spin-independent couplings of light boson fields to electrons and neutrons. The idea is to extract constraints from bounds on nonlinearities in a King plot comparison [16] of isotope shifts of two narrow transitions [17]. We develop a new formalism to interpret these measurements in the context of searching for new low-mass force carriers and propose several elements and transitions that can be used for such analyses. We recast existing measurements into bounds and provide an estimation for the sensitivity of future measurements; see Fig. 1. The validity of our method to bound NP does not rely on a knowledge of the SM contributions to King plot nonlinearities. Its constraining power, however, is limited by the size of the observed nonlinearities. In a case in which King linearity is established at the current state-of-the-art experimental precision-and barring cancellation between the SM and NP contributions-worldrecord sensitivity in a certain mass range will be achieved. For an application of such IS bounds to various models beyond the SM, see Ref. [18].

Factorization of isotope shifts.-Consider an atomic transition, $i$, between narrow atomic states. The difference in the transition frequency $\nu_{i}$ when comparing the isotopes 


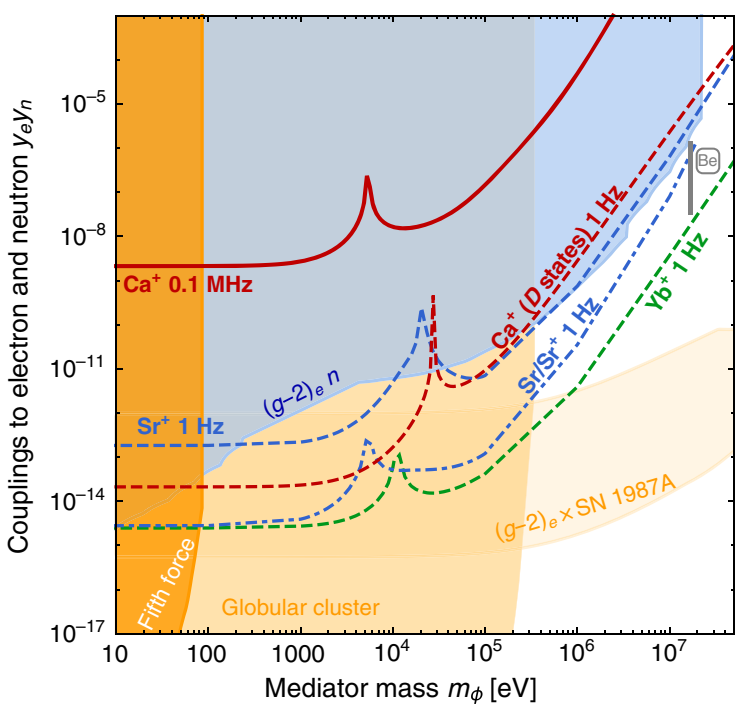

FIG. 1. Limits on the electron and neutron couplings $\left(y_{e} y_{n}\right)$ of a new boson of mass $m_{\phi}$ (for the experimental accuracies $\sigma_{i}$ specified on the labels). Constraint from existing IS data: $\mathrm{Ca}^{+}$ (397 vs $866 \mathrm{~nm}$ [19], the solid red line). IS projections (the dashed lines) for $\mathrm{Ca}^{+}\left(S \rightarrow D\right.$ transitions), $\mathrm{Sr}^{+}, \mathrm{Sr} / \mathrm{Sr}^{+}$, and $\mathrm{Yb}^{+}$. For comparison, existing constraints from other experiments are shown as shaded areas: fifth force (dark orange) [20,21], $(g-2)_{e}$ [22,23] combined with neutron scattering (light blue) [24-27] or SN 1987A (light orange) [28], and star cooling in globular clusters (orange) [29-33]. The gray line at $17 \mathrm{MeV}$ indicates the $y_{e} y_{n}$ values required to accommodate the Be anomaly [34,35].

$A$ and $A^{\prime}$ is the IS, $\nu_{i}^{A A^{\prime}} \equiv \nu_{i}^{A}-\nu_{i}^{A^{\prime}}$. The IS can be phenomenologically written as

$$
\nu_{i}^{A A^{\prime}}=K_{i} \mu_{A A^{\prime}}+F_{i} \delta\left\langle r^{2}\right\rangle_{A A^{\prime}}+\cdots,
$$

where the two terms represent the mass shift and the field shift (FS), respectively $[16,36] ; \mu_{A A^{\prime}} \equiv m_{A}^{-1}-m_{A^{\prime}}^{-1}$, where $m_{A}$ and $m_{A^{\prime}}$ are the masses of isotopes $A$ and $A^{\prime}$, and $\delta\left\langle r^{2}\right\rangle_{A A^{\prime}}$ is dominated by the difference in the charge radii of the two nuclei. Both $\mu_{A A^{\prime}}$ and $\delta\left\langle r^{2}\right\rangle_{A A^{\prime}}$ are purely nuclear quantities that do not depend on the electronic transition $i$, whereas $K_{i}$ and $F_{i}$ are isotope-independent, transitiondependent parameters. Given two electronic transitions, $i=1,2$, one obtains the following linear relation $[16,36]$ :

$$
m \nu_{2}^{A A^{\prime}}=K_{21}+F_{21} m \nu_{1}^{A A^{\prime}}
$$

with $m \nu_{i}^{A A^{\prime}} \equiv \nu_{i}^{A A^{\prime}} / \mu_{A A^{\prime}}, m \delta\left\langle r^{2}\right\rangle_{A A^{\prime}} \equiv \delta\left\langle r^{2}\right\rangle_{A A^{\prime}} / \mu_{A A^{\prime}}, F_{21} \equiv$ $F_{2} / F_{1}$, and $K_{21} \equiv K_{2}-F_{21} K_{1}$.

The formulas in our treatment of NP are simplified by introducing a geometrical description of the above leadingorder (LO) factorization, as King linearity is equivalent to the coplanarity of the vectors. For each transition $i$, we can form a vector $\overrightarrow{m \nu}_{i} \equiv\left(m \nu_{i}^{A A_{1}^{\prime}}, m \nu_{i}^{A A_{2}^{\prime}}, m \nu_{i}^{A A_{3}^{\prime}}\right)$. The nuclear parameters of the field and mass shifts, $\mu_{A A^{\prime}}$ and $\delta\left\langle r^{2}\right\rangle_{A A^{\prime}}$, can also be written as the vectors $\overrightarrow{m \mu} \equiv(1,1,1)$ and $\overrightarrow{m \delta\left\langle r^{2}\right\rangle}$ in the same space, and hence Eq. (1) becomes $\overrightarrow{m \nu}_{i}=K_{i} \overrightarrow{m \mu}+F_{i} \overrightarrow{m \delta\left\langle r^{2}\right\rangle}$. In this language, LO factorization implies that $\overrightarrow{m \nu}_{i}$ must lie in the plane that is defined by $\overrightarrow{m \mu}$ and $\overrightarrow{m \delta\left\langle r^{2}\right\rangle}$, as illustrated in the Supplemental Material [37]. Like King linearity, coplanarity is a purely data-driven test of $\mathrm{LO}$ factorization since it is independent of theoretical input. A change in $K_{i}$ and $F_{i}$ will merely change the direction of $\overrightarrow{m \nu}_{1}$ and $\overrightarrow{m \nu}_{2}$ within the plane, but the qualitative statement of coplanarity remains. In this vector language we can provide a compact expression for a nonlinearity measure,

$$
\mathrm{NL}=\frac{1}{2}\left|\left(\overrightarrow{m \nu}_{1} \times \overrightarrow{m \nu}_{2}\right) \cdot \overrightarrow{m \mu}\right| .
$$

In terms of the King plot, NL is the area of the triangle spanned by the three points shown in the Supplemental Material [37]. Equivalently, in the geometrical picture, it is half the volume of the parallelepiped defined by $\overrightarrow{m \nu}_{1,2}$ and $\overrightarrow{m \mu}$. A given data set is considered linear if $\mathrm{NL}$ is smaller than its first-order propagated error $\sigma_{\mathrm{NL}}=\sqrt{\Sigma_{k}\left(\partial \mathrm{NL} / \partial O_{k}\right)^{2} \sigma_{k}^{2}}$, where the sum runs over all measured observables $O_{k}$ (modified frequency shifts and isotope masses) with standard deviations $\sigma_{k}$.

New physics and violation of King linearity.-We now include a NP contribution by adding a third, also factorized, term to Eq. (1),

$$
\nu_{i}^{A A^{\prime}}=K_{i} \mu_{A A^{\prime}}+F_{i} \delta\left\langle r^{2}\right\rangle_{A A^{\prime}}+\alpha_{\mathrm{NP}} X_{i} \gamma_{A A^{\prime}} ;
$$

namely, $X_{i}$ depends on the form of the NP potential and on the electronic transition, while $\gamma_{A A^{\prime}}$ depends only on the nuclear properties. The parameter $\alpha_{\mathrm{NP}}$ is the NP coupling constant which we would like to probe.

For short-range NP (shorter than the nuclear size), the electronic parameters $X_{i}$ are proportional to those of the FS, $X_{i} \propto F_{i}$. In this case the NP term can be absorbed by redefining $\delta\left\langle r^{2}\right\rangle_{A A^{\prime}}$. Also, if the new physics couples to electrons and nuclei according to their electric charge, $\gamma_{A A^{\prime}}=0$ [38]. However, a long-range force with couplings not proportional to the electric charge (and barring an accidental cancellation) can be severely constrained by tests of King linearity.

Equation (2) written in vectorial form becomes

$$
\overrightarrow{m \nu}_{2}=K_{21} \overrightarrow{m \mu}+F_{21} \overrightarrow{m \nu}_{1}+\alpha_{\mathrm{NP}} \vec{h} X_{1}\left(X_{21}-F_{21}\right),
$$

where $\vec{h}$ is the NP vector in reduced frequency units; that is, $h_{A A^{\prime}} \equiv \gamma_{A A^{\prime}} / \mu_{A A^{\prime}}$ and $X_{21} \equiv X_{2} / X_{1}$. Consequently, NP can lead to a deviation from coplanarity if and only if (i) the new force is not short-range, $X_{21} \neq F_{21}$, and (ii) $\vec{h}$ is not aligned with any linear combination of $\overrightarrow{m \mu}, \overrightarrow{m \nu}_{1}$, or $\overrightarrow{m \nu}_{2}$. 
By solving the set of equations (4), one finds an expression for $\alpha_{\mathrm{NP}}$ that is needed to yield a particular data set $\left\{\overrightarrow{m \nu}_{1}, \overrightarrow{m \nu}_{2}, \overrightarrow{m \mu}\right\}$,

$$
\alpha_{\mathrm{NP}}=\frac{\left(\overrightarrow{m \nu}_{1} \times \overrightarrow{m \nu}_{2}\right) \cdot \overrightarrow{m \mu}}{(\overrightarrow{m \mu} \times \vec{h}) \cdot\left(X_{1} \overrightarrow{m \nu}_{2}-X_{2} \overrightarrow{m \nu}_{1}\right)},
$$

assuming that NP is the dominant contribution to nonlinearity. If linearity holds, then $\alpha_{\mathrm{NP}} \lesssim \sigma_{\alpha_{\mathrm{NP}}}=$ $\sqrt{\Sigma_{k}\left(\partial \alpha_{\mathrm{NP}} / \partial O_{k}\right)^{2} \sigma_{k}^{2}}$. Hence, the sensitivity to $\alpha_{\mathrm{NP}}$ is lost in the limit where the denominator in Eq. (6) vanishes because the NP contribution to nonlinearity is

$$
\mathrm{NL}_{\mathrm{NP}}=\frac{\alpha_{\mathrm{NP}}}{2}(\overrightarrow{m \mu} \times \vec{h}) \cdot\left(X_{1} \overrightarrow{m \nu}_{2}-X_{2} \overrightarrow{m \nu}_{1}\right) .
$$

The presented method of limiting $\alpha_{\mathrm{NP}}$, Eq. (6), contains theory input only in $X_{i}$ and $h_{A A^{\prime}}$, which describe how NP affects the IS. The SM contribution in the factorized limit is fully parametrized by the observables $\vec{\nu}_{i}$ and $\vec{\mu}$. The form of $h_{A A^{\prime}}$ depends on the assumed couplings of new physics to nuclei. For example, if the new interaction couples to quarks, then we expect that $h_{A A^{\prime}} \propto A A^{\prime}[17,39]$. The atomic transition-dependent factors $X_{1,2}$ can be determined by many-body simulation (see below).

Hence, in this method the background is estimated from data and only the NP contribution relies on theory input. This resembles the data-driven background estimation in collider searches for NP. As a consequence, precise predictions of the considered frequencies do not represent a selection criterion for suitable systems.

Thus far, most measurements of IS between spin-zero isotopes have been consistent with King linearity (see, however, the case of samarium in Ref. [40]). Nevertheless, some level of nonlinearity is expected to arise from SM higher-order contributions [41-44]. These contributions, which are related to nuclear physics and electronic-structure dynamics linked together, are presently not understood in a quantitative manner for many-electron systems. One possible source of nonlinearities is of the form of a field shift that depends on the isotope mass. Precision calculations recently showed that this effect is of $O\left(10^{-3}-10^{-4}\right)$ relative to the LO FS in light atoms [45]. Likewise, such contributions in heavier elements with $Z=20-87$ [43], but only for $S \rightarrow P$ transitions, are estimated to be of similar order. Hence, matching the precision of future measurements motivates the calculation of the remaining higher-order corrections.

If a deviation from King linearity is observed, it will be difficult to distinguish the NP and SM contributions to the nonlinearity. In this case there are two options in which further insight on NP can be obtained. The first requires that the status of King nonlinearity calculations would advance and enable us to subtract the SM contributions, and in the process possibly gain new insight on the nature of nuclear effects in IS. To add to that, since nonlinearity in the case of NP is universal and in the case of SM specific to particular atomic configurations, a comparison between measurements in different systems will be beneficial. The second relies on the fact that NP forces are of a longer range than nuclear effects, which require an overlap of the electronic wave function with the nucleus. Hence, it might be possible to identify an observable that is less affected by the nucleus but is still sensitive to the presence of longrange NP interactions. In this regard, IS measurements involving highly excited, so-called Rydberg states might provide a smoking gun for the above types of NP.

For the proposed method to be effective, the element and the specific transitions should be chosen carefully. First, to make significant progress compared to the current precision, we consider narrow optical clock transitions. The most accurate frequency measurements to date, with a relative error of $10^{-18}$, have been performed on such transitions in laser-cooled atoms or ions [46-51]. Second, since the hyperfine interaction of electrons with the nucleus is a source for King nonlinearity [41], we consider only isotopes without nuclear spin.

Contribution of new bosons to isotope shifts.-Next, we discuss how theoretical IS predictions are modified in the presence of hypothetical new force carriers of spin $s=0,1$, or 2 and mass $m_{\phi}$ which couple to electrons and neutrons with strengths $y_{e}$ and $y_{n}$, respectively. The effective spinindependent potential mediated by such bosons between the nucleus and its bound electrons is $V_{\phi}(r)=$ $-\alpha_{\mathrm{NP}}(A-Z) e^{-m_{\phi} r} / r$, where $\alpha_{\mathrm{NP}}=(-1)^{s} y_{e} y_{n} / 4 \pi$. Note that NP could also couple to protons, though without affecting the linearity of the King plot; hence, we neglect such a coupling here. For concreteness, we consider $h_{A A^{\prime}}=$ $A A^{\prime}$ amu for the NP contribution in Eq. (4).

The electronic NP coefficient $X_{i}$ can be determined via many-body calculations [52-58] at lowest order by calculating the overlap of the wave functions with the NP potential; see the Supplemental Material [37] for details.

We identify three regions of the NP interaction range, separated by the electron wave function size, $a_{0} /\left(1+n_{e}\right)$, and the nuclear charge radius, $r_{N} \sim A^{1 / 3} \times(200 \mathrm{MeV})^{-1}$. Here, $a_{0} \approx(4 \mathrm{keV})^{-1}$ is the Bohr radius and $n_{e}$ is the ionization number. For $m_{\phi} \lesssim\left(1+n_{e}\right) / a_{0}$, the "massless limit," the interaction range is larger than the atomic size and $V_{\phi} \propto 1 / r$, so $X_{i}$ becomes independent of $m_{\phi}$. For intermediate masses, $\left(1+n_{e}\right) / a_{0} \lesssim m_{\phi} \lesssim 1 / r_{N}$, the interaction range is within the size of the electron wave function, and the potential $V_{\phi} \propto e^{-m_{\phi} r} / r$ is mass dependent. Hence, detailed knowledge of the electronic wave functions is necessary to evaluate the effect of NP. In the heavy mass limit, $m_{\phi} \gtrsim 1 / r_{N}$, the interaction range is shorter than the nuclear radius and $V_{\phi} \propto \delta(r) /\left(m_{\phi}^{2} r^{2}\right)$. In this limit, the $\mathrm{NP}$ and nuclear charge-radius effects are approximately aligned since $X_{i} \propto F_{i} \propto\left|\Psi_{b}(0)\right|^{2}-\left|\Psi_{a}(0)\right|^{2}$. This results 
in a suppressed sensitivity for NP which scales as $\left(X_{21}-F_{21}\right) \rightarrow 0$; see the above and Ref. [17].

In the massless limit, $X_{i}$ can be estimated without a detailed computation of the atomic wave functions, as in this case the effective potential is Coulomb-like and thus its effects are approximately accounted for by a shift of the fine-structure constant $\alpha$; see the Supplemental Material [37] and Refs. [59,60]. We do not estimate the bounds on $\alpha_{\mathrm{NP}}$ in the heavy mass limit, as in this limit NP effects are indistinguishable from those of finite nuclear size. Bounds are therefore suppressed by a factor of $O\left(r_{N} / a_{0}\right)$.

Current bounds and projections.-Here, we derive the constraints on the product of electron and neutron coupling, $y_{e} y_{n}$, from existing IS data of $\mathrm{Ca}^{+}$and project the bounds for different transitions in alkalilike systems in the $10 \mathrm{eV}-50 \mathrm{MeV}$ mass range, assuming that better IS data will be available in the future. Our results are summarized in Fig. 1 and Table I.

We apply our method to the available IS data of $\mathrm{Ca}^{+}$ (the solid line of Fig. 1). In the massless-boson limit, $m_{\phi} \lesssim 10 \mathrm{keV}$, the bound is essentially independent of $m_{\phi}$. At the high mass limit, we expect that $F_{21}=X_{21}$. Since the theoretical control of $F_{21}$ is worse than the experimental error, one can get an incorrect $m_{\phi}$ dependence for the $y_{n} y_{e}$ bound at that limit. However, the ratio $F_{21}^{\text {th }} / X_{21}\left(F_{21}^{\text {th }}\right.$ is the theoretical value calculated in the absence of NP) has a much smaller error. Thus, in order to account for the reduction in sensitivity as $m_{\phi}$ increases, we rescale the $y_{e} y_{n}$ bound by $\left(1-F_{21}^{\mathrm{exp}} / X_{21}\right) /\left(1-F_{21}^{\mathrm{th}} / X_{21}\right)$, where $F_{21}^{\mathrm{exp}}$ is the measured value of $F_{21}$. We verified with the program Grasp2K [58] that this factor does not change by more than a few percent if the charge radius is changed by order 1 . (Since the latter is known to a few percent accuracy, this is a rather conservative approach.) Indeed, we see that for $m_{\phi}>Z \alpha m_{e}$ the limits get weak, and the sensitivity decreases approximately as $m_{\phi}^{-3}$ for large masses. In the Supplemental Material [37], we give two heuristic arguments that obtain this asymptotic scaling of our loss of sensitivity: the first is based on a perturbative approximation of $X_{i}$, and the second is based on a nonrelativistic QED effective theory [61-63].

TABLE I. The 95\% C.L. bounds on $y_{e} y_{n}$ for a massless mediator $\phi$ from $\mathrm{Ca}^{+}$data [19] and 95\% C.L. projections for $\mathrm{Ca}^{+}, \mathrm{Sr}^{+}, \mathrm{Sr} / \mathrm{Sr}^{+}$, and $\mathrm{Yb}^{+}$assuming on error of $\sigma_{i}=1 \mathrm{~Hz}$.

\begin{tabular}{lcccc}
\hline \hline & $\begin{array}{c}\text { Transition 1 } \\
(\mathrm{nm})\end{array}$ & $\begin{array}{c}\text { Transition 2 } \\
(\mathrm{nm})\end{array}$ & $\begin{array}{c}\text { Accuracy } \\
\sigma_{i}\end{array}$ & $\begin{array}{c}y_{e} y_{n} \text { Bound } \\
\left(m_{\phi}=0\right)\end{array}$ \\
\hline $\mathrm{Ca}^{+}$ & 397.0 & 866.5 & $0.1 \mathrm{MHz}$ & $2 \times 10^{-9}$ \\
$\mathrm{Ca}^{+}$ & 729.3 & 732.6 & $1 \mathrm{~Hz}$ & $2 \times 10^{-14}$ \\
$\mathrm{Sr}^{+}$ & 674.0 & 687.0 & $1 \mathrm{~Hz}$ & $2 \times 10^{-13}$ \\
$\mathrm{Sr}_{\mathrm{Sr}}{ }^{+}$ & 698.4 & 674.0 & $1 \mathrm{~Hz}$ & $3 \times 10^{-15}$ \\
$\mathrm{Yb}^{+}$ & 435.5 & 466.9 & $1 \mathrm{~Hz}$ & $2 \times 10^{-15}$ \\
\hline \hline
\end{tabular}

For current bounds, we consider $\mathrm{Ca}^{+}(Z=20)$. There are five zero-nuclear-spin, stable, or long-lived isotopes with $A=40,42,44,46,48$. References $[19,64]$ reported IS measurements for three isotope pairs $\left(A^{\prime}=42,44,48\right.$ relative to $A=40$ ) in three dipole-allowed transitions in $\mathrm{Ca}^{+}$at wavelengths of $397.0 \mathrm{~nm}\left(S_{1 / 2}-P_{1 / 2}\right), 866.5 \mathrm{~nm}$ $\left(D_{3 / 2}-P_{1 / 2}\right)$ and $393 \mathrm{~nm}\left(S_{1 / 2}-P_{3 / 2}\right.$, not used here) with an uncertainty of $O(100) \mathrm{kHz}$.

Among alternative experiments that probe the $m_{\phi}-y_{e} y_{n}$ parameter space, we consider here only the ones most sensitive to new light bosons coupled to electrons and neutrons. The shaded regions in Fig. 1 summarize the current reach of these experiments. We stress, however, that some of them are derived involving further theoretical assumptions, in contrast to our method, which relies on few theoretical inputs. For new bosons lighter than a few $\times 100 \mathrm{eV}$, fifth-force experiments [20,21] are potentially sensitive. Since the interaction range covered by these experiments is much larger than the atomic size, only forces with nonzero atomic coupling can be probed. For illustration, we show in Fig. 1 the fifth-force bound applicable to $\mathrm{U}(1)_{B-L}$ gauge bosons $[18,65]$.

Furthermore, separately, $y_{n}$ is constrained by various neutron-scattering experiments [24-27], and $y_{e}$ by the anomalous magnetic moment of the electron $(g-2)_{e}$ $[22,23]$ and by electron beam-dump experiments for $m_{\phi}>1 \mathrm{MeV}$.

Both $y_{e}$ and $y_{n}$ are also severely constrained by globular cluster energy loss for masses $m_{\phi}<350 \mathrm{keV}$ [28-33] down to $y_{e} y_{n}<10^{-25}$ and $y_{e}$ by sun cooling [66,67]. Couplings to nucleons in the $10^{-10}-10^{-7}$ range for $m_{\phi} \lesssim 100 \mathrm{MeV}$ may be also excluded by energy loss in the core of the supernova SN 1987A [28,68]. In order to derive an upper bound on $y_{e} y_{n}$, we combine for each mass the best constraint on $y_{n}$ from neutron experiments with $y_{e}$ either from $(g-2)_{e}$ or from astrophysics.

As the precision of optical spectroscopy continues to improve, higher accuracy IS measurements in different systems can be achieved in the near future. Accordingly, we estimate the sensitivity that would be achieved for several transitions in alkali or alkali-earth ions or atoms, given the improved accuracy. Here, we consider a comparison between the two fine-structure split electric quadrupole transitions in $\mathrm{Ca}^{+}$and $\mathrm{Sr}^{+}$. A comparison between the optical clock transitions in $\mathrm{Sr}^{+}$and $\mathrm{Sr}$ and the quadrupole and octupole transitions in $\mathrm{Yb}^{+}$is also presented. In principle, to enhance the sensitivity of our method, it is desirable to compare transitions that involve levels that are as different as possible. For this reason, comparing the two fine-structure split electric quadrupole transitions in $\mathrm{Ca}^{+}$or $\mathrm{Sr}^{+}$is not ideal, especially when compared to the sensitivity of the two considered lines in $\mathrm{Yb}^{+}$or comparing the $\mathrm{E} 2$ line in $\mathrm{Sr}^{+}$with the intercombination line in $\mathrm{Sr}$; see Table I. We include these transitions in our projections 
since their high-resolution IS measurement is experimentally simpler.

All of the transitions above are expected to be measured with $1 \mathrm{~Hz}$ accuracy. Under the assumption that King linearity will hold in future measurements and following the Supplemental Material [37], the projected bounds are plotted in Fig. 1 (the dashed lines) as a function of $m_{\phi}$ and summarized in Table I. The resonance structures at around the $10 \mathrm{keV}$ scale arise from cancellations in the denominator of Eq. (6). These local losses of sensitivity at different masses per atomic system provide another motivation for IS measurements in complementary systems for a good coverage of the parameter space.

The various projections with $1 \mathrm{~Hz}$ accuracy significantly improve the bounds in the $m_{\phi} \geq 10 \mathrm{keV}$ region in parameter space. For lower $m_{\phi}$ values, they are weaker than astrophysical bounds. However, astrophysical bounds are model dependent (for example, the chameleon effect [69]) and are subject to large uncertainties. Thus, an independent laboratory bound in this low-mass region is, nevertheless, worthwhile. For $m_{\phi} \sim$ a few $\mathrm{MeV}$, the projections of $\mathrm{Ca}^{+}$( $S \rightarrow D$ transitions) and $\mathrm{Sr}^{+}$are comparable to the $y_{e} y_{n}$ ones from neutron scattering [25] and $(g-2)_{e}$. Since neutron experiments are affected by uncertainties [27, 70-72], such as those involving the electron-neutronscattering length, the nuclear input values, and the missing higher-order terms in the neutron-scattering cross section, the bounds in the high-mass range well above the neutron energies of $E_{n}<10 \mathrm{keV}$ [25] should be understood as an indication of the order of magnitude. Consequently, theoretically, cleaner IS probes at the same order will already improve the bound robustness. Note that a $\mathrm{Sr} / \mathrm{Sr}^{+}$ $\left(\mathrm{Yb}^{+}\right)$IS comparison would become more effective than other existing methods in probing new bosons above $\sim 10 \mathrm{keV}$, already with $100 \mathrm{~Hz}(1 \mathrm{kHz})$ accuracy (the bound related to $\mathrm{Sr} / \mathrm{Sr}^{+}$constructed from a comparison of transitions involving neutral and ion systems suffers from some numerical instabilities for masses above $20 \mathrm{MeV}$ and is thus not shown). Finally, the range of $y_{e} y_{n}$ needed to explain the Be anomaly $[34,35]$ can be probed by future IS measurements of $\mathrm{Yb}^{+}$at the $1 \mathrm{~Hz}$ level.

We thank J. Feng, O. Firstenberg, I. Galon, R. Hill, S. G. Karshenboim, K. Pachucki, G. Paz, C. Peset, M. Pospelov, P. O. Schmidt, and T. Tait for the fruitful discussions. C. D. and Y.S. are particularly thankful to J. Ekman and P. Jönsson for their help regarding the Grasp2K program. The work of D. B. is supported by the Deutsche Forschungsgemeinschaft (DFG) Reinhart Koselleck project, and the European Research Council (ERC) (Dark-OST Advanced Project). The work of C. D. is supported by the "Investissements d'avenir, Labex ENIGMASS." The work of C.G. is supported by the European Commission through Marie Curie Career Integration Grant No. 631962 and by the Helmholtz
Association through the recruitment initiative program. The work of R.O. is supported by grants from the Israeli Research Foundation (ISF), I-Core, ERC, Minerva, and the Crown Photonics Center. The work of G. P. is supported by grants from the United States-Israel Binational Science Foundation (BSF), ERC, ISF, Minerva, and the Weizmann-UK Making Connections Programme. The work of Y.S. is supported by the U.S. Department of Energy (DOE) under Grant Contracts No. DE-SC-00012567 and No. DE-SC-00015476.

\footnotetext{
*julian.berengut@unsw.edu.au

†budker@uni-mainz.de

cedric.delaunay@lapth.cnrs.fr

§v.flambaum@unsw.edu.au

"claudia.frugiuele@weizmann.ac.il

『elina.fuchs@weizmann.ac.il

** christophe.grojean@desy.de

roni@fnal.gov

咕roee.ozeri@weizmann.ac.il

§ilad.perez@weizmann.ac.il

"IIIsoreqy@mit.edu
}

[1] J. Baron et al. (ACME Collaboration), Order of magnitude smaller limit on the electric dipole moment of the electron, Science 343, 269 (2014).

[2] D. Hanneke, S. Fogwell, and G. Gabrielse, New Measurement of the Electron Magnetic Moment and the Fine Structure Constant, Phys. Rev. Lett. 100, 120801 (2008).

[3] R. Bouchendira, P. Cladé, S. Guellati-Khélifa, F. Nez, and F. Biraben, New Determination of the Fine Structure Constant and Test of the Quantum Electrodynamics, Phys. Rev. Lett. 106, 080801 (2011).

[4] C. Wood, S. Bennett, D. Cho, B. Masterson, J. Roberts, C. Tanner, and C. Wieman, Measurement of parity nonconservation and an anapole moment in cesium, Science 275, 1759 (1997).

[5] J. Guéna, M. Lintz, and M. A. Bouchiat, Measurement of the parity violating $6 S-7 S$ transition amplitude in cesium achieved within $2 \times 10^{-13}$ atomic-unit accuracy by stimulated-emission detection, Phys. Rev. A 71, 042108 (2005).

[6] M. S. Safronova, D. Budker, D. DeMille, D. F. Jackson Kimball, A. Derevianko, and C. W. Clark, Search for New Physics with Atoms and Molecules, arXiv:1710.01833.

[7] S. G. Karshenboim, Precision physics of simple atoms: QED tests, nuclear structure and fundamental constants, Phys. Rep. 422, 1 (2005).

[8] J. S. M. Ginges and V. V. Flambaum, Violations of fundamental symmetries in atoms and tests of unification theories of elementary particles, Phys. Rep. 397, 63 (2004).

[9] J.E. Kim, Light pseudoscalars, particle physics and cosmology, Phys. Rep. 150, 1 (1987).

[10] P. W. Graham, D. E. Kaplan, and S. Rajendran, Cosmological Relaxation of the Electroweak Scale, Phys. Rev. Lett. 115, 221801 (2015).

[11] R. S. Gupta, Z. Komargodski, G. Perez, and L. Ubaldi, Is the relaxion an axion?, J. High Energy Phys. 02 (2016) 166. 
[12] T. Flacke, C. Frugiuele, E. Fuchs, R. S. Gupta, and G. Perez, Phenomenology of relaxion-Higgs mixing, J. High Energy Phys. 06 (2017) 050.

[13] J. L. Feng, T. Moroi, H. Murayama, and E. Schnapka, Third generation familons, $B$ factories, and neutrino cosmology, Phys. Rev. D 57, 5875 (1998).

[14] F. Wilczek, Axions and Family Symmetry Breaking, Phys. Rev. Lett. 49, 1549 (1982).

[15] G. B. Gelmini, S. Nussinov, and T. Yanagida, Does nature like Nambu-Goldstone bosons?, Nucl. Phys. B219, 31 (1983).

[16] W. H. King, Comments on the article peculiarities of the isotope shift in the samarium spectrum, J. Opt. Soc. Am. 53, 638 (1963).

[17] C. Delaunay, R. Ozeri, G. Perez, and Y. Soreq, Probing atomic Higgs-like forces at the precision frontier, Phys. Rev. D 96, 093001 (2017).

[18] C. Frugiuele, E. Fuchs, G. Perez, and M. Schlaffer, Constraining new physics models with isotope shift spectroscopy, Phys. Rev. D 96, 015011 (2017).

[19] F. Gebert, Y. Wan, F. Wolf, C. N. Angstmann, J. C. Berengut, and P. O. Schmidt, Precision Isotope Shift Measurements in Calcium Ions Using Quantum Logic Detection Schemes, Phys. Rev. Lett. 115, 053003 (2015).

[20] M. Bordag, U. Mohideen, and V. M. Mostepanenko, New developments in the Casimir effect, Phys. Rep. 353, 1 (2001).

[21] M. Bordag, G. L. Klimchitskaya, U. Mohideen, and V. M. Mostepanenko, Advances in the Casimir Effect, International Series of Monographs on Physics Vol. 145 (Oxford University, New York, 2009), p. 1.

[22] C. Patrignani et al. (Particle Data Group), Review of particle physics, Chin. Phys. C 40, 100001 (2016).

[23] D. Hanneke, S. Fogwell Hoogerheide, and G. Gabrielse, Cavity control of a single-electron quantum cyclotron: Measuring the electron magnetic moment, Phys. Rev. A 83, 052122 (2011)

[24] R. Barbieri and T.E. O. Ericson, Evidence against the existence of a low mass scalar boson from neutron-nucleus scattering, Phys. Lett. 57B, 270 (1975).

[25] H. Leeb and J. Schmiedmayer, Constraint on Hypothetical Light Interacting Bosons from Low-Energy Neutron Experiments, Phys. Rev. Lett. 68, 1472 (1992).

[26] Yu. N. Pokotilovski, Constraints on new interactions from neutron scattering experiments, Phys. At. Nucl. 69,924 (2006).

[27] V. V. Nesvizhevsky, G. Pignol, and K. V. Protasov, Neutron scattering and extra short range interactions, Phys. Rev. D 77, 034020 (2008).

[28] G. Raffelt, Limits on a $C P$-violating scalar axion-nucleon interaction, Phys. Rev. D 86, 015001 (2012).

[29] W. M. Yao et al. (Particle Data Group), Review of particle physics, J. Phys. G 33, 1 (2006).

[30] J. A. Grifols and E. Masso, Constraints on finite range baryonic and leptonic forces from stellar evolution, Phys. Lett. B 173, 237 (1986).

[31] J. A. Grifols, E. Masso, and S. Peris, Energy loss from the Sun and red giants: Bounds on short range baryonic and leptonic forces, Mod. Phys. Lett. A 04, 311 (1989).

[32] J. Redondo and G. Raffelt, Solar constraints on hidden photons re-visited, J. Cosmol. Astropart. Phys. 08 (2013) 034.
[33] E. Hardy and R. Lasenby, Stellar cooling bounds on new light particles: Plasma mixing effects, J. High Energy Phys. 02 (2017) 033.

[34] J. L. Feng, B. Fornal, I. Galon, S. Gardner, J. Smolinsky, T. M. P. Tait, and P. Tanedo, Protophobic Fifth-Force Interpretation of the Observed Anomaly in ${ }^{8} \mathrm{Be}$ Nuclear Transitions, Phys. Rev. Lett. 117, 071803 (2016).

[35] J. L. Feng, B. Fornal, I. Galon, S. Gardner, J. Smolinsky, T. M. P. Tait, and P. Tanedo, Particle physics models for the $17 \mathrm{MeV}$ anomaly in beryllium nuclear decays, Phys. Rev. D 95, 035017 (2017).

[36] W. H. King, Isotope Shifts in Atomic Spectra (Plenum Press, New York, 1984).

[37] See Supplemental Material at http://link.aps.org/ supplemental/10.1103/PhysRevLett.120.091801 for a visualization of the vector space.

[38] J. Jaeckel and S. Roy, Spectroscopy as a test of Coulomb's law: A probe of the hidden sector, Phys. Rev. D 82, 125020 (2010).

[39] H. E. Haber, G. L. Kane, and T. Sterling, The fermion mass scale and possible effects of Higgs bosons on experimental observables, Nucl. Phys. B161, 493 (1979).

[40] J. A. R. Griffith, G. R. Isaak, R. New, and M. P. Ralls, Anomalies in the optical isotope shifts of samarium, J. Phys. B 14, 2769 (1981).

[41] C. W. P. Palmer and D. N. Stacey, Theory of anomalous isotope shifts in samarium, J. Phys. B 15, 997 (1982).

[42] E. C. Seltzer, $K$ x-ray isotope shifts, Phys. Rev. 188, 1916 (1969).

[43] S. A. Blundell, P. E. G. Baird, C. W. P. Palmer, D. N. Stacey, and G. K. Woodgate, A reformulation of the theory of field isotope shift in atoms, J. Phys. B 20, 3663 (1987).

[44] G. Torbohm, B. Fricke, and A. Rosén, State-dependent volume isotope shifts of low-lying states of group-II $a$ and -II $b$ elements, Phys. Rev. A 31, 2038 (1985).

[45] M. Puchalski and K. Pachucki, Nuclear structure effects in the isotope shift with halo nuclei, Hyperfine Interact. 196, 35 (2010).

[46] B. J. Bloom, T. L. Nicholson, J. R. Williams, S. L. Campbell, M. Bishof, X. Zhang, W. Zhang, S. L. Bromley, and J. Ye, An optical lattice clock with accuracy and stability at the $10^{-18}$ level, Nature (London) 506, 71 (2014).

[47] T. Rosenband, D. Hume, P. Schmidt, C. Chou, A. Brusch, L. Lorini, W. Oskay, R. Drullinger, T. Fortier, J. Stalnaker et al., Frequency ratio of $\mathrm{Al}^{+}$and $\mathrm{Hg}^{+}$single-ion optical clocks; metrology at the 17th decimal place, Science $\mathbf{3 1 9}$, 1808 (2008).

[48] P. Dubé, A. A. Madej, Z. Zhou, and J. E. Bernard, Evaluation of systematic shifts of the ${ }^{88} \mathrm{Sr}^{+}$single-ion optical frequency standard at the $10^{-17}$ level, Phys. Rev. A 87, 023806 (2013).

[49] M. Chwalla, J. Benhelm, K. Kim, G. Kirchmair, T. Monz, M. Riebe, P. Schindler, A. Villar, W. Hänsel, C. Roos et al., Absolute Frequency Measurement of the ${ }^{40} \mathrm{Ca}^{+} 4 s^{2} S_{1 / 2}-3 d^{2} D_{5 / 2}$ Clock Transition, Phys. Rev. Lett. 102, 023002 (2009).

[50] C. Tamm, S. Weyers, B. Lipphardt, and E. Peik, Stray-fieldinduced quadrupole shift and absolute frequency of the ${ }^{688-\mathrm{THz}}{ }^{171} \mathrm{Yb}^{+}$single-ion optical frequency standard, Phys. Rev. A 80, 043403 (2009). 
[51] N. Huntemann, C. Sanner, B. Lipphardt, C. Tamm, and E. Peik, Single-Ion Atomic Clock with $3 \times 10^{-18}$ Systematic Uncertainty, Phys. Rev. Lett. 116, 063001 (2016).

[52] V. A. Dzuba, V. V. Flambaum, and M. G. Kozlov, Combination of the many-body perturbation theory with the configuration-interaction method, Phys. Rev. A 54, 3948 (1996).

[53] J. C. Berengut, V. A. Dzuba, and V. V. Flambaum, Isotope shift calculations for atoms with one valence electron, Phys. Rev. A 68, 022502 (2003).

[54] J. C. Berengut, V. V. Flambaum, and M. G. Kozlov, Calculation of isotope shifts and relativistic shifts in C I, C II, C III, and C IV, Phys. Rev. A 73, 012504 (2006).

[55] W. R. Johnson, S. A. Blundell, and J. Sapirstein, Finite basis sets for the Dirac equation constructed from $B$ splines, Phys. Rev. A 37, 307 (1988).

[56] V. M. Shabaev, I. I. Tupitsyn, V. A. Yerokhin, G. Plunien, and G. Soff, Dual Kinetic Balance Approach to Basis-Set Expansions for the Dirac Equation, Phys. Rev. Lett. 93, 130405 (2004).

[57] J. C. Berengut, Particle-hole configuration interaction and many-body perturbation theory: Application to $\mathrm{Hg}^{+}$, Phys. Rev. A 94, 012502 (2016).

[58] P. Jonsson, G. Gaigalas, J. Biero, C. F. Fischer, and I. Grant, New version: Grasp2K relativistic atomic structure package, Comput. Phys. Commun. 184, 2197 (2013).

[59] D. Budker, D. DeMille, E. D. Commins, and M. S. Zolotorev, Experimental investigation of excited states in atomic dysprosium, Phys. Rev. A 50, 132 (1994).

[60] N. Leefer, L. Bougas, D. Antypas, and D. Budker, Towards a new measurement of parity violation in dysprosium, arXiv:1412.1245.
[61] W. E. Caswell and G. P. Lepage, Effective Lagrangians for bound state problems in QED, QCD, and other field theories, Phys. Lett. 167B, 437 (1986).

[62] R. J. Hill, G. Lee, G. Paz, and M. P. Solon, NRQED Lagrangian at order 1/M4 , Phys. Rev. D 87, 053017 (2013).

[63] T. Kinoshita and M. Nio, Radiative corrections to the muonium hyperfine structure. 1. The $\alpha^{2}(Z \alpha)$ correction, Phys. Rev. D 53, 4909 (1996).

[64] C. Shi, F. Gebert, C. Gorges, S. Kaufmann, W. Nörtershäuser, B. K. Sahoo, A. Surzhykov, V. A. Yerokhin, J. C. Berengut, F. Wolf, J. C. Heip, and P. O. Schmidt, Unexpectedly large difference of the electron density at the nucleus in the $4 p^{2} P_{1 / 2,3 / 2}$ fine-structure doublet of $\mathrm{Ca}^{+}$, Appl. Phys. B 123, 2 (2017).

[65] R. Harnik, J. Kopp, and P. A. N. Machado, Exploring $\nu$ signals in dark matter detectors, J. Cosmol. Astropart. Phys. 07 (2012) 026.

[66] J. Redondo, Helioscope bounds on hidden sector photons, J. Cosmol. Astropart. Phys. 07 (2008) 008.

[67] J. Jaeckel and A. Ringwald, The low-energy frontier of particle physics, Annu. Rev. Nucl. Part. Sci. 60, 405 (2010).

[68] K. Blum and D. Kushnir, Neutrino signal of collapse-induced thermonuclear supernovae: The case for prompt black hole formation in SN 1987A, Astrophys. J. 828, 31 (2016).

[69] C. Burrage and J. Sakstein, A compendium of chameleon constraints, J. Cosmol. Astropart. Phys. 11 (2016) 045.

[70] F. Wissmann, M. Schumacher, and M. I. Levchuk, On approaches to measure the electromagnetic polarizabilities of the neutron, Eur. Phys. J. A 1, 193 (1998).

[71] I. Antoniadis et al., Short-range fundamental forces, C.R. Phys. 12, 755 (2011).

[72] D. Tucker-Smith and I. Yavin, Muonic hydrogen and MeV forces, Phys. Rev. D 83, 101702 (2011). 